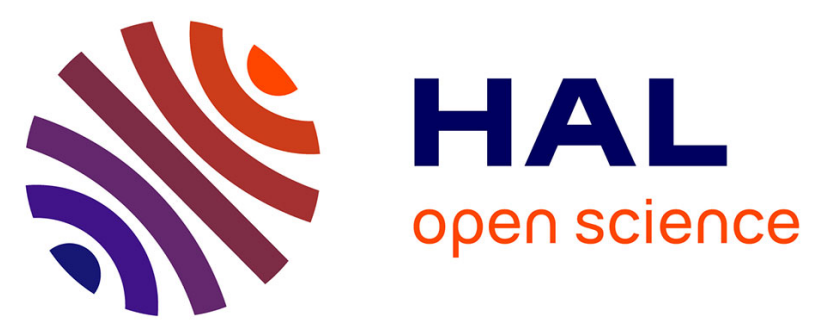

\title{
Metagenomic approaches for investigating the role of the microbiome in gut health and inflammatory diseases.
} Rodrigo Carvahlo, Filipe Carmo, Sara Heloisa, Barbara Cordeiro, Aline Vaz, Enrico Gimenez, Luis Goulart, Aristóteles Góes-Neto, Yves Le Loir, Gwénaël Jan, et al.

\section{To cite this version:}

Rodrigo Carvahlo, Filipe Carmo, Sara Heloisa, Barbara Cordeiro, Aline Vaz, et al.. Metagenomic approaches for investigating the role of the microbiome in gut health and inflammatory diseases.. Metagenomics for Gut Microbes, INTECH EUROPE, 2018, 978-1-78923-110-6. 10.5772/intechopen.68370 . hal-01793728

\section{HAL Id: hal-01793728 \\ https://hal.science/hal-01793728}

Submitted on 16 May 2018

HAL is a multi-disciplinary open access archive for the deposit and dissemination of scientific research documents, whether they are published or not. The documents may come from teaching and research institutions in France or abroad, or from public or private research centers.
L'archive ouverte pluridisciplinaire HAL, est destinée au dépôt et à la diffusion de documents scientifiques de niveau recherche, publiés ou non, émanant des établissements d'enseignement et de recherche français ou étrangers, des laboratoires publics ou privés. 


\title{
Metagenomic Approaches for Investigating the Role of the Microbiome in Gut Health and Inflammatory Diseases
}

\author{
Rodrigo Carvalho, Fillipe Carmo, Sara Heloisa, \\ Barbara Cordeiro, Aline Vaz, Enrico Gimenez, \\ Luis Goulart, Aristóteles Góes-Neto, Yves Le Loir, \\ Gwénaël Jan and Vasco Azevedo
}

Additional information is available at the end of the chapter

http://dx.doi.org/10.5772/intechopen.72031

\begin{abstract}
The human gut microbiota makes fundamental contributions to host metabolism and immune system. Therefore, perturbations in its composition, a process known as dysbiosis, have an important role in the development of several chronicle diseases, mainly intestinal inflammatory disorders. Culture-independent molecular methods are allowing scientific community to uncover substantive findings, thus giving a more detailed description of the human intestinal microbiota. This chapter presents a review on current metagenomic approaches, based on next-generation sequencing technologies, for investigating bacterial taxonomic classification and predictive function associated with the human gut in health and disease. In this context, we describe recent studies that have been trying to elucidate important alterations in microbiome composition across individuals according to delivery mode, aging, diet and medication that might be linked to susceptibility to immune-mediated diseases. A description of the main bacterial taxa and genes acting in dysbiosis during inflammation, focusing on chronic inflammatory bowel diseases and colorectal cancer, is also explored in this chapter.
\end{abstract}

Keywords: gut microbiota, metagenomics, dysbiosis, inflammatory bowel diseases, colorectal cancer

\section{Introduction}

Microbiota is considered as an essential organ of humans and other animals, which carry out many functions that host cells cannot. A major portion of these mutualistic microbes is found 
in the human gut, where they occupy niches that make contributions to nutrient processing, pathogen colonization resistance and mucosal immune system development [1]. The intestinal microbiome is formed by hundreds of different bacterial species colonizing mucosal surfaces. Its compositional structure differs across human populations according to geographic regions in the world method of delivery at childbirth, breast or bottle feeding, age, diet and medications [2]. Actually, the role of one individual microbiota is composed by the repertoire of expressed genes, known as metagenome. Impressively, it is estimated that humans possess 10 million extra genes from intestinal bacteria [3]. Significant perturbation of the gut microbiota can lead to a dysbiosis state, which compromise important functions in host immunity and raise susceptibility to immune-mediated diseases [4]. Therefore, there has been great interest in identifying the metagenomic content of the gut microbiota which can be used to treat or prevent diseases. In this context, extensive endeavor are being carried out to elucidate the gut ecosystem and molecular mechanisms underlying the pathogenesis of several intestinal disorders [5]. Culture-independent methods, in particular next-generation sequencing technologies, have prompted a huge breakthrough in our knowledge regarding the microbial communities colonizing the human body and their functional beneficence to host health [6].

\section{Metagenomic sequencing approaches for investigating intestinal microbiomes}

\subsection{Bacterial identification by $16 \mathrm{~S}$ rRNA gene sequencing}

New generation sequencing technologies are capable of processing high amount of DNA in a relative short time using $16 \mathrm{~S}$ ribosomal (16S rRNA) genetic information. Several high throughput platforms such as 454 Roche GS FLX, Applied Biosystems SOLiD System, Illumina HiSeq and MiSeq System and Ion Torrent Personal Genome Machine (PGM) have been used for this kind of metagenomic approach $[7,8]$. The molecular-based taxonomic investigation for bacteria employs direct sequencing of PCR-amplified small sequences of 16S rRNA gene from extracted DNA, generally using universal primers annealing conserved nucleotides to amplify one or more fragments of variable regions. As a few numbers of base pairs can change in a very short period of evolutionary time, amplicons around $300 \mathrm{bp}$ are frequently enough for taxonomic assignment [9]. The sequences at a pre-defined level of identity stand for grouped clusters of similar sequencing reads, known as Operational Taxonomic Unit (OTU), which corresponds to a group of very similar 16S sequences. Reference databases (GreenGenes, myRDP, NCBI) are used to classify OTUs providing identification of taxonomy, relative frequencies and diversity of community composition in samples obtained from the certain ecosystem [10,11]. This approach allows identification of new species and investigation of low-abundance bacteria and even uncultivated gut microbial communities from a single analysis. In addition, these technologies are faster and more accurate compared to classical identification methods (cloning and culture) [12]. However, this approach has some limitations regarding information about the microbiome function, mainly because several species of bacteria have not been characterized yet and secondly due to a great variability found among individuals, it is expected that the microbiota function present high redundancy, in which different species may occupy the same niche in the gut [13]. 


\subsection{Shotgun sequencing for predictive functional analyses}

The whole metagenome sequencing can be performed using a shotgun approach. In such genomic survey strategy, multiple continuous overlapping sequences (contigs), which are assembled from fragmented sequences and obtained from total purified genomic DNA, are used for identifying genes through alignment with bacterial reference genomes and databases (KEGG, SEED and NCBI) [6]. Shotgun approach is quite versatile, in which the samples can be submitted to various methods, including nebulization, endonucleases, or sonication for random fragmentation of DNA, sequencing a subsequent contig assembly and annotation. Furthermore, advanced computational methods applying different algorithms are frequently being developed for more accurate assembly and annotation of genes, thus allowing functional characterization in complex environments like the human gut [14, 15]. This method also provides identification of variants and polymorphisms and gives a more comprehensive understanding on the functional information of microorganism communities, for example, by reconstructing metabolic pathways in silico [12]. A major limitation of this strategy is that metagenomic sequencing of multiple individuals is extremely expensive in comparison with $16 \mathrm{~S}$ rRNA sequencing and generation of a large amount of data demands intense computational analysis, most of time to be performed by bioinformatics specialists [16].

\subsection{Metagenomic consortium}

Massive increase in the amount of data from human gut microbiota and identification of genes or families of genes submitted in the databases has prompted the creation of consortia such as the Human Microbiome Project in healthy individuals, which led to establishment of a reference microbial genome database according to results of 165 profiling of 242 healthy adults from the United States [17]. The European milestone Metagenomics of the Human Intestinal Tract (MetaHIT) intended to identify potential links between the association of gut microbiome with obesity and inflammatory bowel disease (IBD) from $540 \mathrm{~Gb}$ of DNA from stool samples of 124 healthy or sick individuals [18]. Moreover, about 1000 bacterial species were found and each individual in this study is estimated to contain at least 160 species, and in addition, 18 species of bacteria were common to 124 individuals [19]. Nevertheless, complementary approaches to metagenomic studies as well as integrative analysis are required to understand the complex and intrinsic interactions with gut microbiota and hosts, like metatranscriptomics, metaproteomics for studying the functional aspects of the microbiota and metabolomics [20, 21].

\section{The gut microbiome in health}

\subsection{Nutritional and metabolic functions}

Gut microbiota is essential for the host digestion and nutrition, as they can produce unique nutrients from indigestible substrates [22]. For instance, many polysaccharides, which are found in vegetables from our diet, such as cellulose, xylans, resistant starch and inulin, are digested by certain species that colonize the intestines. Metagenomic studies revealed that the capacity to digest xyloglucans found in onion and lettuce is a specific trait of Bacteroides sp. [23]. Through 
a mutualistic relationship, the digestion of non-digestible compounds by microbiota may yield energy for microbial growth and end products such as short-chain fatty acids (SCFAs). SCFAs, mainly acetate, propionate and butyrate, are an important energy source for the colonic epithelium and as a key factor for regulating pro-inflammatory immune responses in the gut. Acetate and propionate are important for the liver and peripheral tissues as well, because they act as substrates for metabolic functions such as gluconeogenesis and lipogenesis [5]. The capacity of gut microbiomes metabolic activity, when regarding SCFAs production, may depend on how much complex carbohydrates are ingested through our diet and on the composition of the gut microbiota as well [24].

A study using a model of gnotobiotic mice demonstrated that fermentation of dietary fructans increases when animals colonized with Bacteroides thetaiotaomicron are co-colonized with Methanobrevibacter smithii. The first species produces more acetate and formate, whereas the second uses formate for methanogenesis, illustrating the importance of interaction between the microbiota organisms to promote nutrient fermentation and absorption and consequently metabolic functions $[25,26]$.

The ingestion of prebiotics such as inulin can promote expansion of Faecalibacterium prausnitzii and Bifidobacterium sp. in humans. Both species are important for metabolic functions and immune response regulation in the intestinal mucosa as they produce butyrate and folate, respectively. Folate can be synthesized by the large bowel microbiota and is essential for the synthesis of precursors of nucleic acids, contribute to epigenetic effects, and amino acid metabolism. For example, B. bifidum and B. longum produce folate in high concentrations [27, 28].

Vitamin B12 is synthesized by different bacteria, for example, Propionibacterium freudenreichii and Lactobacillus reuteri [29]. Vitamin B12 and its corrinoid precursors play an important role in the gut microbiota as $80 \%$ of human intestinal microbes express transporters to capture corrinoids and use vitamin B12 as a cofactor for metabolic pathways. For example, B. thetaiotaomicron encodes three vitamin B12 acquisition systems. Folate and vitamin B12 also serve as regulators of gene expression in human gut bacteria and might control genomic interactions between the microbiota and host [30].

\subsection{Immune system regulation and resistance against opportunistic pathogens colonization}

The mucosal immune system is responsible for maintaining gut homeostasis as it must remain vigilant against pathogen infections while limiting over inflammatory responses against the majority of commensal organisms that comprise the microbiota. Symbiotic bacteria play an important role in preventing inflammatory diseases, however in dysbiosis, opportunistic pathogens may stimulate a local immune response resulting a tissue damage. Therefore, the microbiota has the potential to exert both pro-inflammatory and anti-inflammatory responses, meaning that the balance in the composition of gut microbiome may be intrinsically involved in the proper function of the immune system [31, 32]. Many bacterial species from the gut microbiota such as Lactobacillus sp. and Bifidobacterium sp. have the ability to activate molecular 
mechanism on several cell types constituting the epithelial barrier function, such as specialized epithelial cells like goblet cells and paneth cells [33,34]. Goblet cells are the main cells involved in mucus production, which is considered the first line of host defense against pathogens. Current studies reveal that several commensal microbes can stimulate Muc2 expression, a major component of the intestinal mucus [35].

Enterocytes, such as paneth cells, which reside at the base of the intestinal crypts, are specialized in producing antimicrobial peptides preventing overstimulation of the immune system by keeping commensal bacteria $50 \mu \mathrm{m}$ apart from the small intestinal epithelial surface [35]. Studies suggest that expression of these antimicrobial peptides, including RegIII $\gamma$ and defensins, is driven by the microbiota. Certain components of the microbiota, including $B$. thetaiotaomicron, confer resistance to Candida albicans by promoting the expression of $\mathrm{H} 1 \mathrm{~F}-1 \alpha$, a transcriptional regulator that induces the expression of the antimicrobial peptide LL-37, with anti-Candida activity [36].

Microbiota induction of the adaptive responses of the immune system, including B cells and $\mathrm{T}$ cells, plays a central role in the defense against intestinal pathogens and regulation of inflammation in the gut. Certain intestinal bacterial species play a major role in the differentiation of $\mathrm{T}$ cells into different subsets, including $\mathrm{T}$ helper cells Th1s, Th2s, Th17s and T regulatory cells (Tregs) [35]. The development and functionality of Tregs in the gut depend on the presence of specific commensal microbes. Administration of a pool containing bacterial strains from the Clostridia clusters IV, XIVa and XVIII has been shown to restore the population of Tregs to those observed in conventional mice. One mechanism by which Clostridia species, including F. prausnitzii, may enhance Treg differentiation is through production of SCFA [37]. Similarly, vitamin A metabolite retinoic acid, whose production has been shown to be partly controlled by the microbiota, also supports anti-inflammatory function [38].

Production of IgA by B cells is a key factor for the host to control infections on mucosal surfaces, including the gastrointestinal tract. Several studies have demonstrated that the intestinal microbiota influences B-cell development and antibody production. The gut microbiota affects IgA class-switch recombination in either T-cell-independent or dependent pathways. T-cell-independent produces IgA with low affinity but directed towards the microbiota. Bacteria that colonize the epithelial surface of the intestinal mucosa, such as Mucispirillum, Clostridium scindens and Akkermansia muciniphila which are segmented filamentous bacteria, can activate t-cell-dependent pathway and regulation of mucosal IgA responses $[39,40]$.

The microbiota can also confer resistance to pathogens by directly inhibiting them, without the involvement of the gut immune system. Certain commensal strains can produce and secrete small molecules with bacteriostatic or bactericidal activity, such as bacteriocins or microcins produced by Gram-positive and Gram-negative species, respectively. SCFA can also influence the expression of virulence factors. For example, butyrate and propionate can downregulate the expression of pathogenicity island 1 (SP1) genes in Salmonella typhimurium, which is crucial for this bacterium to invade intestinal epithelial cells. A major mechanism by 
which the microbiota inhibits intestinal colonization by bacterial pathogens is through nutrient competition [35].

\subsection{Microbiome composition in human ontogeny}

The gut microbiome assumes different characteristics regarding diversity, structure and functional gene repertoires over human lifetime (Figure 1). From the compositional perspective, microbiota becomes more and more complex with time, along with periods of ecological stability and fluctuation due to new environmental expositions, until it reaches a dynamic equilibrium in adulthood [13].

\subsubsection{Birth}

It is commonly accepted by scientific community that our first exposition to microbes may occur at birth delivery. However, recent metagenomics studies reveal that non-harmful bacteria may colonize the placenta, amniotic fluid and other fetal components from healthy term pregnancies [41]. The bacterial taxa identified in the fetal environment present low abundance but are diverse, including many species associated with the oral and intestinal microbiomes, such as Fusobacterium spp. and Bacteroides spp. Moreover, similar bacterial communities can be found in meconium, suggesting that the colonization of the gut may start with intrauterine resident bacteria [42]. Nevertheless, the elucidation of the probable mechanism in which those bacteria translocate to the gut of the fetus is required to sustain the hypothesis of prenatal colonization. Regarding the gut microbiome just after birth, it has significantly lower diversity and higher variability among individuals compared to the microbiome in adulthood [24]. The dominant bacteria at phylum level in the neonatal gut include Firmicutes, Proteobacteria and Actinobacteria with lower levels of Bacteroidetes, a dominant phylum in the adult gut microbiome [43].

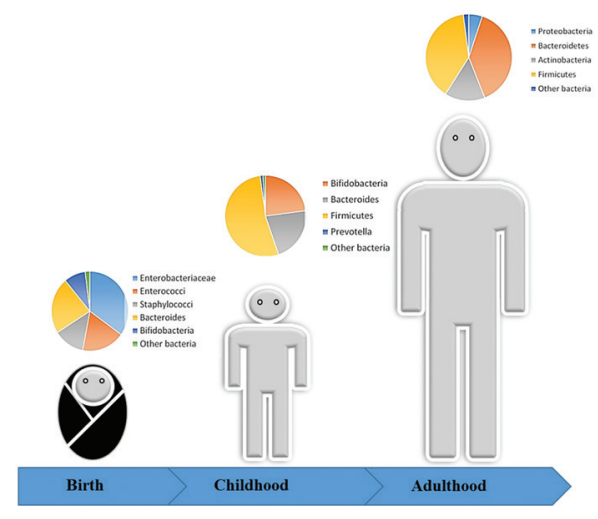

Figure 1. Intestinal microbiota in birth, childhood and adulthood. Birth delivery starts the colonization of the gut, thought the caesarian mode can alter the microbiome composition in newborns. In childhood, gut microbiota shows increased diversity due to introduction of solid foods. In adulthood, the gut microbiota achieves stability but several lifestyle factors may influence the microbiome composition, such as diet, physical activities, alcohol, smoking, drugs and hygiene. 


\subsubsection{Mode of delivery}

The modes of delivery significantly influence the neonatal microbiome [44]. In vaginally delivered infants, the gut is primarily colonized by bifidobacteria as well as lactobacilli and enterobacteria. When compared to children delivered in natural terms, the microbiomes of caesarian-delivered individuals show lower diversity and lower abundance of these bacterial taxa [45]. Moreover, infants delivered by cesarean section have more Escherichia coli as well as Clostridium difficile [46]. A recent study has been trying to evaluate whether cesarean-delivered infants exposure to maternal vaginal fluids are able to colonize them with the natural microbiota that is supposed to be acquired during passage through the birth canal. However, these findings revealed that such inoculation provides minimal effect on the bacterial community of the infants, which presented fewer species and lower levels of Lactobacillus spp. and Bacteroides spp. comparing with samples originally obtained from vaginally delivered infants [47]. It is important to reiterate that despite of discrepancies between studies in the literature, most of them show that mode of delivery may select certain bacterial groups over other types.

\subsubsection{Childhood}

Previously, based on classical microbiological studies, it was thought that the human gut microbiome would achieve a stable status during the first 4 years of life. However, contemporary studies using metagenomic approaches have shown a different composition in childhood regarding the human gut microbiota when compared to adulthood [48, 49]. In American children cohorts, a study demonstrated that they present higher frequencies of Firmicutes, Proteobacteria and Actinobacteria and less Bacteroidetes [50]. Moreover, teenager's microbiomes seem to be enriched in butyrate-producing bacteria such as Alistipes spp., B. vulgatus and B. xylanisolvens and Roseburia spp., Faecalibacterium spp., Ruminococcus spp., that seems to play anti-inflammatory roles [51]. Also, on a nutritional function perspective, microbiomes of children contain more vitamin B12-producing bacterial species than adults [52].

Although many functional features of the microbiome of children remain to be profoundly investigated, in general, it is known that childhood microbiome represents a dynamic ecosystem, which probably has an impact on health later in life. In this context, several environmental exposures are supposed to be associated with differences found among individuals. These factors include drugs exposure, contact with domestic animals, hygiene, geography and diet [52]. Metagenomic studies suggest that diet has a determinant role for driving the development of childhood microbiome as it needs to adapt to different conditions of nutrients availability [53].

\subsubsection{Breast feeding versus bottle feeding}

In the first years of life, the human gut microbiome is richer in genes involved in digestion of oligosaccharides found in breast milk, while in later childhood, due to the ingestion of solid foods, the gut metagenome is richer in genes involved in the digestion of polysaccharides and vitamin biosynthesis. Therefore, different microbe exposure in modes of feeding might significantly influence microbiome composition and function [52, 53]. Breast-fed children 
show an increase in Actinobacteria and a decrease in Firmicutes and Proteobacteria, whereas bottle-fed ones exhibit more abundance of potential pathogens such as E. coli and C. difficile $[42,54]$. Interestingly, breast milk contains many compounds that might affect the microbiome composition of the infant gut in a positive way, such as immunoglobulin, prebiotic oligosaccharides and diverse maternal milk microbiota species that continually colonize the infant gut [55]. The majority of the studies show that the composition of the gut microbiota in breast-fed infants is enriched in aerobic organisms compared to formula-fed ones, which present higher prevalence of anaerobic and facultative anaerobic organisms such as Bacteroides sp. Certain Bacteroides sp. strains are able to digest milk oligosaccharides, suggesting a potential competitive relationship between Bifidobacterium sp. and Bacteroides sp. in breast-fed infants. Metagenomic findings have been important for providing new advances regarding translational researches such as the development of infant formulas that are more similar in composition and function to breast milk. In this context, Oligosaccharide-enriched formulas have been developed, favoring the colonization of infants gut by greater numbers of bifidobacteria [43].

\subsubsection{Adulthood}

Healthy adult humans may harbor more than 1000 species of bacteria belonging to different bacterial phyla with Bacteroidetes and Firmicutes being the dominant ones [56]. The microbiota of adults can achieve the highest diversity regarding human ontogeny development. There is also considerable variation in the intestinal environment, compared to other sites of the gut, and among healthy individuals. The proportion of each phylum apparently varies according to geographical distribution $[2,57]$. For instance, some studies have shown that Firmicutes are more prevalent in adults in rural communities, whereas adults in industrialized societies seem to present higher levels of Bacteroidetes [58]. A higher Firmicutes to Bacteroidetes ratio has been mostly associated with metabolism function and body weight gain, although further investigation is required to shed light on the species-associated role in healthy individuals cohort across different geographical location to understand their influence in leanness/obesity [24]. In general, the gut microbiome in adulthood remains relatively stable through adulthood, except following perturbations such as pathogen infections, antibiotic drugs or drastic dietary shifts [13]. However, as we age, the gut microbiome is enriched in more traits associated with inflammation and metabolic dysfunction. A reduction in Bacteroides spp., Prevotella spp. and F. prausnitzii and an increase in Enterobacteriaceae have been associated with an overall decrease in the quality of life in old age [51]. In general, the gut microbiome in the elder adults exhibits a higher ratio of Firmicutes to Bacteroidetes when compared to young adults and reduction in symbiotic microbes such as Bifidobacteria and Bacteroides [59].

\section{Dysbiosis in the pathogenesis of inflammatory bowel diseases}

As described above in this chapter, substantial changes in microbiota may sometimes lead to dysfunction resulting in a dysbiosis state. This process has an important role in diseases involving inflammatory responses in the gut, for example, in inflammatory bowel diseases 
(IBDs) such as Crohn's disease (CD) and ulcerative colitis (UC). Moreover, excessive oxidative long-term activity of IBD is associated with colorectal cancer (CRC) (Figure 2) [60]. IBDs have been considered as global health problem [61], affecting around 3.1 million people in the USA $[62,63]$. CD and UC are chronic diseases characterized by periods of relapse and remission. Clinical symptoms of both diseases are similar to each other; however, UC activity is restricted to the colon while CD may affect any part of the gut, more frequently in the ileum and proximal colon [64].

It has been reported that patients with IBD have a reduced diversity of gut microbiota, characterized by the depletion of commensal species belonging to the phylum Firmicutes and Bacteroidetes [65, 66]. Metagenomic studies have pointed out a decreasing in short-chain fatty acids (SCFAs) metabolism genes such as butyrate, which play an important role in the maturation of regulatory T cells, in the gut microbiota of IBD patients [67]. Moreover, it was observed that patients have a reduction in a number of many SCFA-producing species from Clostridia groups, remarkably F. prausnitzii $[68,69]$. Microbiota patterns such as the reduction in F. prausnitzii have been consistently associated with CD patients, during period of active disease or remission, from different geographical regions including Europe and South Asia and may thus serve as a reliable clinical marker [70, 71]. The reduction in species from Lactobacillus and Bifidobacterium genus has an important role in IBD pathogenesis as well, as these bacteria can downregulate the expression of key proinflammatory cytokines and chemokines in the gut [70]. In the other hand, opportunistic commensals, such as Mycobacterium avium sub. paratuberculosis, C. difficile, Ruminococcus gnavus and enterobacteria, are increased [19]. Commensal Sulfate-reducing bacteria are considered as a key factor in the initiation and maintenance of IBD as these bacteria reduce disulfide bonds of the mucus barrier, thereby allowing exposure of the host cells to pathogenic bacteria and toxins [72]. Therefore, these

IBD and the gut microbiota $\quad \mathrm{CRC}$ and the gut microbiota

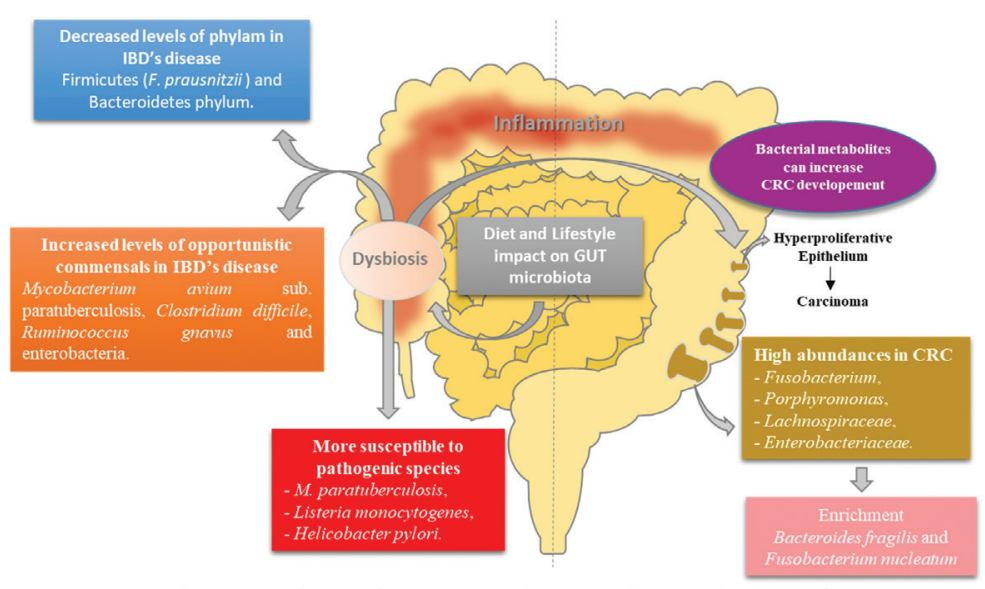

Figure 2. Dysbiosis in pathogenesis of IBD and CRC. Increased levels of metabolites produced by opportunistic commensals of the gut microbiome modulates the effects of inflammation process and cancer tumorigenesis. 
patients develop a certain predisposition for the colonization of facultative pathogens, such as invasive E. coli, that express several virulence factors involved in adhesion and invasion of the epithelial barrier [73, 74]. Moreover, colonization by pathogenic species, such as M. paratuberculosis, Listeria monocytogenes or Helicobacter species, can worsen the symptoms of IBD as these bacteria can activate proinflammatory signaling cascades into the host [75].

Besides bacteria, gut microbiome fungi and virus may also be involved in IBD. Metagenomic studies demonstrated that inflamed guts present high frequency of certain bacteriophages [76, 77]. In regard of fungi, few studies in mice show some yeast species that can inhibit the over growth of certain opportunistic commensals and affect the gut homeostasis. However, in humans, much research is required to elucidate the role of fungi and virus microbiome in the gut [78].

Currently, there is much discussion about the use of fecal transplant for transference of microbiota from healthy individuals to IBD patients as an alternative therapeutic strategy. Promising outcomes have been achieved against $C$. difficile infection in clinical trials, which is often found in the gut of IBD patients [79]. However, in UC clinical trials, the fecal transplant efficacy is controversial $[80,81]$. Regarding CD clinical trials, children cohorts have shown better results with fecal microbiota transplantation than adult cohorts $[82,83]$.

\subsection{Colorectal cancer}

CRC is the most common form of gastrointestinal tract cancer, globally the third leading cause of cancer, and is associated with significant mortality affecting both men and women with 1.4 million people diagnosed annually [60,84].

Different from other kinds of cancer affecting the large bowel which are caused by point mutations in several genes that control cell proliferation, survival, differentiation and migration [60], CRC is mostly attributed to environmental factors and lifestyle such as high fat diet, alcohol, red meat, smoking, obesity, or lack of physical activity [56, 85]. There are several studies showing a connection between inflammatory processes and carcinogenesis, although the contribution of immunological mechanisms and inflammation to malignancy of CRC, it is not fully elucidated [86-88]. Immune cells, cytokines and other mediators of the immune system that are directly influenced by dysbiosis play an important role in the stages of tumorigenesis in the colon, including onset, promotion, progression, and metastasis [86].

Several studies have been trying to identify components of the microbiota that play pivotal roles in inflammation process and in the progression of colorectal cancer by creating microenvironments that favor tumorigenesis development (Table 1). Metagenomic analysis of microbiome provides insights of interactions and contributes to understand how the bacterial species can be related to CRC development, as it has been observed that bacterial populations present in fecal samples are distinct from not only tissue biopsies but also between inter-individual microbial communities, even in samples with the same subtypes of cancer. Usually fecal samples and biopsies are collected for 16S RNA gene sequencing generating results about diversity and abundance of the species making part of the gut microbial community $[89,90]$. 


Main findings
Microbiomes of IBD subjects fluctuate more than those of
healthy individuals
UC and CD have distinct microbiomes. F. prausnitzii and
E. coli were found decreased and increased, respectively, in
CD. These species among others could be used as microbiome
markers to discriminate CD and UC

Microbiome colonizing the mucosa is different between inflamed subjects with CD and UC. At phylum level, Bacteroidetes is more frequent in CD while Proteobacteria and Firmicutes were more frequently observed in UC. At genus level, Faecalibacterium, Bacteroides and Pseudomonas were significantly different between the inflamed CD and UC

The role of the metabolites produced by the microbiota in dysbiosis was correlated to IBD. Dysbiosis characterized by changes in Firmicutes and Proteobacteria phyla.

Decreased levels of Roseburia and Faecalibacterium were found in $\mathrm{CD}$ and $\mathrm{UC}$

Metabolites and fecal microbiome can be useful to discriminate between healthy subjects and patients with IBD. At genus level, Escherichia, Faecalibacterium, Streptococcus, Sutterella and Veillonella were increased, while level of Bacteroides, Flavobacterium and Oscillospira decreased in IBD group

Mucosa-associated dysbiosis was identified in IBD patients. $\mathrm{CD}$ and UC may be distinguishable from the mucosaassociated microbial community structure. CD patients have increased levels of Escherichia, Ruminococcus, Cetobacterium, Actinobacillus and Enterococcus comparing to controls and UC subjects, and a significant decrease in Faecalibacterium, Coprococcus, Prevotella and Roseburia as well

No consistent overrepresentation of potential pathogenic bacteria in CRC tissue

Increased abundance of Coriobacteridae, Roseburia,

Fusobacterium and Faecalibacterium

Decreased abundance of Enterobacteriaceae (Citrobacter, Shigella, Cronobacter, Kluyvera, Serratia and Salmonella spp.)

The gut microbiome could be used as a biomarker for CRC Adenoma: increased abundances of Ruminococcaceae, Clostridium, Pseudomonas and Porphyromonadaceae. Lower abundances of Bacteroides, Lachnospiraceae, Clostridiales and Clostridium

Carcinoma: increased abundances of Fusobacterium, Porphyromonas, Lachnospiraceae and Enterobacteriaceae. Lower abundances of Bacteroides, Lachnospiraceae and Clostridiales

F. nucleatum is prevalent in cases of proximal colon cancer. Amount of F. nucleatum increases linearly along the bowel subsites from rectum to cecum

$\begin{array}{ll}\text { Disease } & \text { Sampling description } \\ \text { IBD } & \begin{array}{l}\text { Fecal samples from } 109 \\ \text { patients with IBD }(\mathrm{CD}, \mathrm{n}=49 ; \\ \\ \mathrm{UC}, \mathrm{n}=60)\end{array} \\ \text { IBD } & \begin{array}{l}2045 \text { non-IBD and IBD fecal } \\ \text { samples from four countries } \\ \text { (Spain, Belgium, the UK and } \\ \text { Germany) }\end{array} \\ & \begin{array}{l}\text { Analysis of the microbiota } \\ \text { composition of ileum, cecum, } \\ \text { IBDid-colon and rectum samples } \\ \text { from } 166 \text { individuals }\end{array}\end{array}$

Ref

[100]

IBD Microbiota of intestinal biopsies and stool samples from 231 IBD and healthy

IBD Microbiota and the metabolites in stool of 183 subjects (UC-82, CD-50 and 51 healthy controls)

IBD 174 mucus samples from 43 UC subjects, 26 CD subjects and 14 non-IBD controls

CRC Resections for primary colon adenocarcinoma of 6 patients

CRC Analysis of fecal samples from healthy individuals, adenoma and carcinoma patients (30 subject for each clinical group) carcinoma cases 


\begin{tabular}{|c|c|c|c|}
\hline Main findings & Disease & Sampling description & Ref \\
\hline $\begin{array}{l}\text { F. nucleatum, B. fragilis and F. prausnitzii could be identified as } \\
\text { useful prognostic biomarkers for CRC } \\
\text { B. fragilis and F. prausnitzii correlated with patient's survival } \\
\text { in CRC } \\
\text { F. nucleatum presented higher abundance in non-survival } \\
\text { group }\end{array}$ & CRC & $\begin{array}{l}\text { Tissues samples from } 108 \\
\text { patients in stages I-IV of CRC } \\
\text { with different prognosis }\end{array}$ & [109] \\
\hline $\begin{array}{l}\text { CRC microbiome is stage-specific and appears to evolve with } \\
\text { disease progression. Enrichment of organisms including } \\
\text { Bacteroides fragilis, Fusobacterium nucleatum and under- } \\
\text { representation of Bacteroides vulgatus, Bacteroides uniformis and } \\
\text { Faecalibacterium prausnitzii } \\
\text { Enrichment of oral pathobionts in poor prognosis tumors } \\
\text { and cancers: Parvimonas micra, Porphyromonas gingivalis and } \\
\text { Prevotella spp. }\end{array}$ & CRC & $\begin{array}{l}\text { Tissue was sampled from } 158 \\
\text { CRC patients, } 24 \text { adenoma } \\
\text { patients and } 14 \text { normal colon } \\
\text { controls }\end{array}$ & [110] \\
\hline $\begin{array}{l}\text { Multiple fusobacteria members did not correlate with } \\
\text { CRC. Enriched F. nucleatum, F. necrophorum, Leptotrichia } \\
\text { trevisanii, B. fragilis, Parvimonas micra, Peptostreptococcus } \\
\text { stomatis and Gemella morbillorum } \\
\text { Low levels of F. varium and Cetobacterium somerae in CRC }\end{array}$ & CRC & $\begin{array}{l}\text { 16S rRNA amplicon sequence } \\
\text { raw datasets from } 12 \text { studies }\end{array}$ & [111] \\
\hline
\end{tabular}

Table 1. Recent metagenomic studies in clinical investigation of intestinal inflammatory diseases.

Considering CRC microenvironment and the functionality of intestinal microbiome, it has a high importance as a risk factor or can be directly associated to CRC. Chronic inflammatory processes driven by dysbiosis can affect all stages of tumor development by compounds that can damage DNA, for example, reactive oxygen species (ROS) promoting CRC development [91]. During an inflammatory response, this microenvironment generates ROS and reactive nitrogen intermediates (RNIs) that lead to deleterious DNA promoting carcinogenesis damage or activating pivotal signaling pathways for adenoma formation and growth [86]. In a study investigating CRC in mouse model of tumorigenesis, it was demonstrated that long-term inflammation-mediated breakdown of protective intestinal barriers promotes the production of some inflammatory cytokines such as IL-17 and IL-23 that lead to tumor growth by facilitating bacterial translocation and consequently microbial products that trigger tumorigenesis resulting in adenoma invasion [92]. Blooms of enterobacteria also seem to play a role in CRC as indicated by metagenomic studies of luminal microbiota of inflamed Il10-/- mice reveal that E. coli can promote cancer activity modulating tumor development once host inflammation has been established [87, 93].

In a study with fecal samples from 74 patients with CRC, the microbiome was enriched in Fusobacterium nucleatum and Peptostreptococcus stomatis. Moreover, co-occurrence of these taxa with the other two, Parvimonas micra and Solobacterium moorei, was found. Interestingly, $P$. micra and the Gram-negative F. nucleatum can induce inflammatory responses by binding to lipopolysaccharides from Gram-negative bacteria. Furthermore, F. nucleatum was shown to increase intestinal tumorigenesis through recruitment of infiltrating immune cells and through activation of $\beta$-catenin signaling [90]. In another study, in which the role of F. nucleatum in CRC was investigated by metagenomic analyses of 511 colorectal carcinomas from Japanese patients, it was identified a significant increase in the occurrence of the bacteria [89]. 
An investigation in 34 patients with four CRC subtypes showed enrichment of Fusobacteria and Bacteroidetes, and decreased levels of Firmicutes and Proteobacteria, including Porphyromonas gingivalis, P. micra, P. stomatis and F. nucleatum, create a particular condition by recruiting $\mathrm{T}$ cells resembling the immunological aspects in specific colorectal tumors [94]. Prorok-Hamon et al. (2014) have shown, by PCR screening, that from 281 E. coli isolates from IBD patients, CRC patients presented increased levels of 21 colonic mucosa-associated E. coli strains, with pathogenic traits, including M-cell translocation, angiogenesis and genotoxicity properties [95].

Some works using metagenomics and metabolomics integrative analyses found that enriched levels of Fusobacteria and Proteobacteria create a specific mucosal metabolic microenvironment that was associated with CRC pathogenesis through a large number of chemical and molecular signaling pathways [96-98]. Although fecal microbiome and metabolome may positively correlate with CRC conditions, the role of such interactions is still poorly understood in the pathogenesis of the disease [96].

Helicobacter pylori which well established in the development of stomach cancer since 1994 being classified as carcinogenic by the International Agency for Research on Cancer has also been pointed out as risk factor for $\mathrm{CRC}$, as the infection leads to initial inflammatory response by stimulating IL-1 $\beta$ production, and consequently causing epithelium injury such as metaplasia [85]. In some studies, there was an indication of increased risk of colorectal adenomas by the presence of $H$. pylori due to hypergastrinemia; however, there is a controversy in other metagenomic studies that did not found correlation or even did not identify the presence of the pathogen in the analyzed samples [99].

\section{Concluding remarks}

These recent metagenomic studies reiterate that microbiome intrinsic factors from particular communities and lifestyle are extremely important and should be considered for future development of novel therapies for IBD. Despite of all efforts to accurately unravel the microbiome role in the gut and its relationship with gut inflammation through metagenomic sequencing approaches, it seems that more complex mechanism might be involved in dysbiosis linked to chronic inflammatory diseases, such as IBD and CRC. In this context, further advances in the area are required to achieve a more precise definition of gut microbiome role, which could allow investments for the search of more effective therapies.

\section{Author details}

Rodrigo Carvalho ${ }^{1 *}$, Fillipe Carmo ${ }^{1}$, Sara Heloisa ${ }^{1}$, Barbara Cordeiro ${ }^{1}$, Aline Vaz ${ }^{1}$, Enrico Gimenez ${ }^{1}$, Luis Goulart ${ }^{2}$, Aristóteles Góes-Neto ${ }^{1}$, Yves Le Loir ${ }^{3}$, Gwénaël Jan ${ }^{3}$ and Vasco Azevedo ${ }^{1}$

*Address all correspondence to: rodrigodoc2@gmail.com

1 Federal University of Minas Gerais (UFMG-ICB), Belo Horizonte, MG, Brazil

2 Federal University of Uberlandia, Uberlandia (UFU-IGB), MG, Brazil

3 INRA-Agrocampus Ouest UMR 1253 STLO, Rennes, France 


\section{References}

[1] Messer JS, Liechty ER, Vogel OA, Chang EB. Evolutionary and ecological forces that shape the bacterial communities of the human gut. Mucosal Immunology. 2017;10:567579. DOI: $10.1038 / \mathrm{mi} .2016 .138$

[2] Rook G, Bäckhed F, Levin BR, McFall-Ngai MJ, McLean AR. Evolution, human-microbe interactions, and life history plasticity. The Lancet. 2017;390:521-530. DOI: 10.1016/ S0140-6736(17)30566-4

[3] Burcelin R. When gut fermentation controls satiety: A PYY story. Molecular Metabolism. 2016;6:10-11. DOI: 10.1016/j.molmet.2016.11.005

[4] Lin L, Zhang J. Role of intestinal microbiota and metabolites on gut homeostasis and human diseases. BMC Immunology. 2017;18:2. DOI: 10.1186/s12865-016-0187-3

[5] Boulangé CL, Neves AL, Chilloux J, Nicholson JK, Dumas M-E. Impact of the gut microbiota on inflammation, obesity, and metabolic disease. Genome Medicine. 2016;8:42. DOI: 10.1186/s13073-016-0303-2

[6] Nagarajan N, Pop M. Sequence assembly demystified. Nature Reviews. Genetics. 2013;14:157-167. DOI: 10.1038/nrg3367

[7] Shendure J, Ji H. Next-generation DNA sequencing. Nature Biotechnology. 2008;26:11351145. DOI: $10.1038 / \mathrm{nbt} 1486$

[8] Fuller CW, Middendorf LR, Benner SA, Church GM, Harris T, Huang X, et al. The challenges of sequencing by synthesis. Nature Biotechnology. 2009;27:1013-1023. DOI: 10.1038/nbt.1585

[9] Cole JR, Chai B, Farris RJ, Wang Q, Kulam-Syed-Mohideen AS, McGarrell DM, et al. The ribosomal database project (RDP-II): Introducing myRDP space and quality controlled public data. Nucleic Acids Research. 2007;35:D169-D172. DOI: 10.1093/nar/gkl889

[10] Handelsman J. Metagenomics: Application of genomics to uncultured microorganisms. Microbiology and Molecular Biology Reviews (MMBR). 2004;68:669-685. DOI: 10.1128/ MMBR.68.4.669-685.2004

[11] Lepage P, Leclerc MC, Joossens M, Mondot S, Blottière HM, Raes J, et al. A metagenomic insight into our gut's microbiome. Gut. 2013;62:146-158. DOI: 10.1136/gutjnl-2011-301805

[12] Tringe SG, von Mering C, Kobayashi A, Salamov AA, Chen K, Chang HW, et al. Comparative metagenomics of microbial communities. Science. 2005;308:554-557. DOI: 10.1126/ science. 1107851

[13] Moya A, Ferrer M. Functional redundancy-induced stability of gut microbiota subjected to disturbance. Trends in Microbiology. 2016;24:402-413. DOI: 10.1016/j.tim.2016.02.002

[14] Kuczynski J, Lauber CL, Walters WA, Parfrey LW, Clemente JC, Gevers D, et al. Experimental and analytical tools for studying the human microbiome. Nature Reviews. Genetics. 2011;13:47-58. DOI: 10.1038/nrg3129 
[15] Thomas T, Gilbert J, Meyer F. Metagenomics - A guide from sampling to data analysis. Microbial Informatics and Experimentation. 2012;2:3. DOI: 10.1186/2042-5783-2-3

[16] Fraher MH, O'Toole PW, Quigley EMM. Techniques used to characterize the gut microbiota: A guide for the clinician. Nature Reviews. Gastroenterology \& Hepatology. 2012;9:312322. DOI: $10.1038 /$ nrgastro.2012.44

[17] Morgan XC, Huttenhower C. Chapter 12: Human microbiome analysis. PLoS Computational Biology. 2012;8:e1002808. DOI: 10.1371/journal.pcbi.1002808

[18] Li J, Jia H, Cai X, Zhong H, Feng Q, Sunagawa S, et al. An integrated catalog of reference genes in the human gut microbiome. Nature Biotechnology. 2014;32:834-841. DOI: 10.1038/nbt.2942

[19] Qin J, Li R, Raes J, Arumugam M, Burgdorf KS, Manichanh C, et al. A human gut microbial gene catalog established by metagenomic sequencing. Nature. 2010;464:59-65. DOI: 10.1038/nature08821

[20] Lee PY, Chin S-F, Neoh H, Jamal R. Metaproteomic analysis of human gut microbiota: Where are we heading? Journal of Biomedical Science. 2017;24:36. DOI: 10.1186/ s12929-017-0342-z

[21] Petriz BA, Franco OL. Metaproteomics as a complementary approach to gut microbiota in health and disease. Frontiers in Chemistry. 2017;5:4. DOI: 10.3389/fchem.2017.00004

[22] Tremaroli V, Bäckhed F. Functional interactions between the gut microbiota and host metabolism. Nature. 2012;489:242-249. DOI: 10.1038/nature11552

[23] Larsbrink J, Rogers TE, Hemsworth GR, McKee LS, Tauzin AS, Spadiut O, et al. A discrete genetic locus confers xyloglucan metabolism in select human gut Bacteroidetes. Nature. 2014;506:498-502. DOI: 10.1038/nature12907

[24] Conlon MA, Bird AR. The impact of diet and lifestyle on gut microbiota and human health. Nutrients. 2014;7:17-44. DOI: 10.3390/nu7010017

[25] Samuel BS, Gordon JI. A humanized gnotobiotic mouse model of host-archaeal-bacterial mutualism. Proceedings of the National Academy of Sciences of the United States of America. 2006;103:10011-10016. DOI: 10.1073/pnas.0602187103

[26] Ríos-Covián D, Ruas-Madiedo P, Margolles A, Gueimonde M. De los Reyes-Gavilán CG, Salazar N. Intestinal short chain fatty acids and their link with diet and human health. Frontiers in Microbiology. 2016;7. DOI: 10.3389/fmicb.2016.00185

[27] Ramirez-Farias C, Slezak K, Fuller Z, Duncan A, Holtrop G, Louis P. Effect of inulin on the human gut microbiota: Stimulation of Bifidobacterium adolescentis and Faecalibacterium prausnitzii. The British Journal of Nutrition. 2009;101:541-550. DOI: 10.1017/S00071 14508019880

[28] Zhao Y, Yu Y-B. Intestinal microbiota and chronic constipation. SpringerPlus. 2016;5. DOI: 10.1186/s40064-016-2821-1 
[29] LeBlanc JG, Milani C, de Giori GS, Sesma F, van Sinderen D, Ventura M. Bacteria as vitamin suppliers to their host: A gut microbiota perspective. Current Opinion in Biotechnology. 2013;24:160-168. DOI: 10.1016/j.copbio.2012.08.005

[30] Biesalski HK. Nutrition meets the microbiome: Micronutrients and the microbiota. Annals of the New York Academy of Sciences. 2016;1372:53-64. DOI: 10.1111/nyas.13145

[31] Sherwin E, Sandhu KV, Dinan TG, Cryan JF. May the force be with you: The light and dark sides of the microbiota-gut-brain Axis in neuropsychiatry. CNS Drugs. 2016; 30:1019-1041. DOI: 10.1007/s40263-016-0370-3

[32] Zmora N, Bashiardes S, Levy M, Elinav E. The role of the immune system in metabolic health and disease. Cell Metabolism. 2017;25:506-521. DOI: 10.1016/j.cmet.2017.02.006

[33] Carmo FLR, Rabah H, Cordeiro BF, Silva SHD, Jan G, Azevedo VA, et al. Applications of Probiotic Bacteria and Dairy Foods in Health. Current Research in Microbiology. 1ed. Wilmington: Open Access eBooks. 2017;1:1-33

[34] Carvalho RDDO, do Carmo FLR, de Oliveira Junior A, Langella P, Chatel J-M, BermúdezHumarán LG, et al. Use of wild type or recombinant lactic acid bacteria as an alternative treatment for gastrointestinal inflammatory diseases: A focus on inflammatory bowel diseases and mucositis. Frontiers in Microbiology. 2017;8. DOI: 10.3389/fmicb.2017.00800

[35] Ubeda C, Djukovic A, Isaac S. Roles of the intestinal microbiota in pathogen protection. Clinical \& Translational Immunology. 2017;6:e128. DOI: 10.1038/cti.2017.2

[36] Fan D, Coughlin LA, Neubauer MM, Kim J, Kim MS, Zhan X, et al. Activation of HIF- $1 \alpha$ and LL-37 by commensal bacteria inhibits Candida albicans colonization. Nature Medicine. 2015;21:808-814. DOI: 10.1038/nm.3871

[37] Atarashi K, Tanoue T, Oshima K, Suda W, Nagano Y, Nishikawa H, et al. Treg induction by a rationally selected mixture of Clostridia strains from the human microbiota. Nature. 2013;500:232-236. DOI: 10.1038/nature12331

[38] Hand TW, Vujkovic-Cvijin I, Ridaura VK, Belkaid Y. Linking the microbiota, chronic disease, and the immune system. Trends in Endocrinology and Metabolism. 2016;27:831843. DOI: 10.1016/j.tem.2016.08.003

[39] Sommer F, Bäckhed F. The gut microbiota-Masters of host development and physiology. Nature Reviews. Microbiology. 2013;11:227-238. DOI: 10.1038/nrmicro2974

[40] Honda K, Littman DR. The microbiota in adaptive immune homeostasis and disease. Nature. 2016;535:75-84. DOI: 10.1038/nature18848

[41] Cao B, Stout MJ, Lee I, Mysorekar IU. Placental microbiome and its role in preterm birth. NeoReviews. 2014;15:e537-e545. DOI: 10.1542/neo.15-12-e537

[42] Arrieta M-C, Stiemsma LT, Amenyogbe N, Brown EM, Finlay B. The intestinal microbiome in early life: Health and disease. Frontiers in Immunology. 2014;5. DOI: 10.3389/ fimmu.2014.00427 
[43] Gritz EC, Bhandari V. The human neonatal gut microbiome: A brief review. Frontiers in Pediatrics. 2015;3. DOI: 10.3389/fped.2015.00017

[44] Mueller NT, Bakacs E, Combellick J, Grigoryan Z, Dominguez-Bello MG. The infant microbiome development: Mom matters. Trends in Molecular Medicine. 2015;21:109-117. DOI: 10.1016/j.molmed.2014.12.002

[45] Neu J, Rushing J. Cesarean versus vaginal delivery: Long term infant outcomes and the hygiene hypothesis. Clinics in Perinatology. 2011;38:321-331. DOI: 10.1016/j.clp.2011. 03.008

[46] Yang I, Corwin EJ, Brennan PA, Jordan S, Murphy JR, Dunlop A. The infant microbiome: Implications for infant health and neurocognitive development. Nursing Research. 2016;65:76-88. DOI: 10.1097/NNR.0000000000000133

[47] Dominguez-Bello MG, De Jesus-Laboy KM, Shen N, Cox LM, Amir A, Gonzalez A, et al. Partial restoration of the microbiota of cesarean-born infants via vaginal microbial transfer. Nature Medicine. 2016;22(3):250. DOI: 10.1038/nm.4039

[48] Houghteling PD, Walker WA. Why is initial bacterial colonization of the intestine important to the infant's and child's health? Journal of Pediatric Gastroenterology and Nutrition. 2015;60:294-307. DOI: 10.1097/MPG.0000000000000597

[49] Rodríguez JM, Murphy K, Stanton C, Ross RP, Kober OI, Juge N, et al. The composition of the gut microbiota throughout life, with an emphasis on early life. Microbial Ecology in Health and Disease. 2015;26. DOI: 10.3402/mehd.v26.26050

[50] Cheng J, Ringel-Kulka T, Heikamp-de Jong I, Ringel Y, Carroll I, de Vos WM, et al. Discordant temporal development of bacterial phyla and the emergence of core in the fecal microbiota of young children. The ISME Journal. 2016;10:1002-1014. DOI: 10.1038/ ismej.2015.177

[51] Hollister EB, Riehle K, Luna RA, Weidler EM, Rubio-Gonzales M, Mistretta T-A, et al. Structure and function of the healthy pre-adolescent pediatric gut microbiome. Microbiome. 2015;3. DOI: 10.1186/s40168-015-0101-x

[52] Greenhalgh K, Meyer KM, Aagaard KM, Wilmes P. The human gut microbiome in health: Establishment and resilience of microbiota over a lifetime. Environmental Microbiology. 2016;18:2103-2116. DOI: 10.1111/1462-2920.13318

[53] Thompson AL, Monteagudo-Mera A, Cadenas MB, Lampl ML, Azcarate-Peril MA. Milkand solid-feeding practices and daycare attendance are associated with differences in bacterial diversity, predominant communities, and metabolic and immune function of the infant gut microbiome. Frontiers in Cellular and Infection Microbiology. 2015;5:1-13. DOI: $10.3389 /$ fcimb.2015.00003

[54] Jandhyala SM, Talukdar R, Subramanyam C, Vuyyuru H, Sasikala M, Reddy DN. Role of the normal gut microbiota. World Journal of Gastroenterology (WJG). 2015;21:87878803. DOI: $10.3748 /$ wjg.v21.i29.8787 
[55] Laforest-Lapointe I, Arrieta M-C. Patterns of early-life gut microbial colonization during human immune development: An ecological perspective. Frontiers in Immunology. 2017;8:1-13. DOI: 10.3389/fimmu.2017.00788

[56] Shreiner AB, Kao JY, Young VB. The gut microbiome in health and in disease. Current Opinion in Gastroenterology. 2015;31:69-75. DOI: 10.1097/MOG.0000000000000139

[57] Lloyd-Price J, Abu-Ali G, Huttenhower C. The healthy human microbiome. Genome Medicine. 2016;8:1-11. DOI: 10.1186/s13073-016-0307-y

[58] De Filippo C, Cavalieri D, Di Paola M, Ramazzotti M, Poullet JB, Massart S, et al. Impact of diet in shaping gut microbiota revealed by a comparative study in children from Europe and rural Africa. Proceedings of the National Academy of Sciences of the United States of America. 2010;107:14691-14696. DOI: 10.1073/pnas.1005963107

[59] Saraswati S, Sitaraman R. Aging and the human gut microbiota-From correlation to causality. Frontiers in Microbiology. 2015;5:1-4. DOI: 10.3389/fmicb.2014.00764

[60] Coleman OI, Nunes T. Role of the microbiota in colorectal cancer: Updates on microbial associations and therapeutic implications. BioResearch Open Access. 2016;5:279-288. DOI: $10.1089 /$ biores.2016.0028

[61] Gismera CS, Aladrén BS. Inflammatory bowel diseases: A disease (s) of modern times? Is incidence still increasing? World Journal of Gastroenterology. 2008;14:5491-5498

[62] Dahlhamer JM, Zammitti EP, Ward BW, Wheaton AG, Croft JB. Prevalence of inflammatory bowel disease among adults aged $\geq 18$ years - United States, 2015. MMWR. Morbidity and Mortality Weekly Report. 2016;65:1166-1169. DOI: 10.15585/mmwr.mm6542a3

[63] Molodecky NA, Soon IS, Rabi DM, Ghali WA, Ferris M, Chernoff G, et al. Increasing incidence and prevalence of the inflammatory bowel diseases with time, based on systematic review. Gastroenterology. 2012;142:46-54.e42; quiz e30. DOI: 10.1053/j.gastro.2011.10.001

[64] Tontini GE, Vecchi M, Pastorelli L, Neurath MF, Neumann H. Differential diagnosis in inflammatory bowel disease colitis: State of the art and future perspectives. World Journal of Gastroenterology (WJG). 2015;21:21-46. DOI: 10.3748/wjg.v21.i1.21

[65] Walker AW, Sanderson JD, Churcher C, Parkes GC, Hudspith BN, Rayment N, et al. High-throughput clone library analysis of the mucosa-associated microbiota reveals dysbiosis and differences between inflamed and non-inflamed regions of the intestine in inflammatory bowel disease. BMC Microbiology. 2011;11:7. DOI: 10.1186/1471-2180-11-7

[66] DeGruttola AK, Low D, Mizoguchi A, Mizoguchi E. Current understanding of Dysbiosis in disease in human and animal models. Inflammatory Bowel Diseases. 2016;22:11371150. DOI: 10.1097/MIB.0000000000000750

[67] Smith PM, Howitt MR, Panikov N, Michaud M, Gallini CA, Bohlooly YM, et al. The microbial metabolites, short-chain fatty acids, regulate colonic Treg cell homeostasis. Science. 2013;341:569-573. DOI: 10.1126/science.1241165 
[68] Sokol H, Pigneur B, Watterlot L, Lakhdari O, Bermúdez-Humarán LG, Gratadoux J-J, et al. Faecalibacterium prausnitzii is an anti-inflammatory commensal bacterium identified by gut microbiota analysis of Crohn's disease patients. Proceedings of the National Academy of Sciences of the United States of America. 2008;105:16731-16736. DOI: 10.1073/pnas.0804812105

[69] Takahashi K, Nishida A, Fujimoto T, Fujii M, Shioya M, Imaeda H, et al. Reduced abundance of butyrate-producing bacteria species in the Fecal microbial Community in Crohn's disease. Digestion. 2016;93:59-65. DOI: 10.1159/000441768

[70] Manichanh C, Borruel N, Casellas F, Guarner F. The gut microbiota in IBD. Nature Reviews. Gastroenterology \&Hepatology.2012;9:599-608. DOI: 10.1038/nrgastro.2012.152

[71] Rehman A, Rausch P, Wang J, Skieceviciene J, Kiudelis G, Bhagalia K, et al. Geographical patterns of the standing and active human gut microbiome in health and IBD. Gut. 2016;65:238-248. DOI: 10.1136/gutjnl-2014-308341

[72] Ijssennagger N, van der Meer R, van Mil SWC. Sulfide as a mucus barrier-breaker in inflammatory bowel disease? Trends in Molecular Medicine. 2016;22:190-199. DOI: 10.1016/j.molmed.2016.01.002

[73] Lodes MJ, Cong Y, Elson CO, Mohamath R, Landers CJ, Targan SR, et al. Bacterial flagellin is a dominant antigen in Crohn's disease. The Journal of Clinical Investigation. 2004;113:1296-1306. DOI: 10.1172/JCI20295

[74] Subramanian S, Roberts CL, Hart CA, Martin HM, Edwards SW, Rhodes JM, et al. Replication of colonic Crohn's disease mucosal Escherichia Coli isolates within macrophages and their susceptibility to antibiotics. Antimicrobial Agents and Chemotherapy. 2008;52:427-434. DOI: 10.1128/AAC.00375-07

[75] Lebeer S, Vanderleyden J, De Keersmaecker SCJ. Host interactions of probiotic bacterial surface molecules: Comparison with commensals and pathogens. Nature Reviews. Microbiology. 2010;8:171-184. DOI: 10.1038/nrmicro2297

[76] Breitbart M, Hewson I, Felts B, Mahaffy JM, Nulton J, Salamon P, et al. Metagenomic analyses of an uncultured viral community from human Feces. Journal of Bacteriology. 2003;185:6220-6223. DOI: 10.1128/JB.185.20.6220-6223.2003

[77] Minot S, Sinha R, Chen J, Li H, Keilbaugh SA, GD W, et al. The human gut virome: Interindividual variation and dynamic response to diet. Genome Research. 2011;21:16161625. DOI: $10.1101 /$ gr.122705.111

[78] Ni J, GD W, Albenberg L, Tomov VT. Gut microbiota and IBD: Causation or correlation? Nature Reviews. Gastroenterology \& Hepatology. 2017;14:573-584. DOI: 10.1038/ nrgastro.2017.88

[79] Gough E, Shaikh H, Manges AR. Systematic review of intestinal microbiota transplantation (fecal bacteriotherapy) for recurrent Clostridium difficile infection. Clinical Infectious Diseases: An Official Publication of the Infectious Diseases Society of America. 2011;53:994-1002. DOI: 10.1093/cid/cir632 
[80] Angelberger S, Reinisch W, Makristathis A, Lichtenberger C, Dejaco C, Papay P, et al. Temporal bacterial community dynamics vary among ulcerative colitis patients after fecal microbiota transplantation. The American Journal of Gastroenterology. 2013;108:16201630. DOI: 10.1038/ajg.2013.257

[81] Suskind DL, Brittnacher MJ, Wahbeh G, Shaffer ML, Hayden HS, Qin X, et al. Fecal microbial transplant effect on clinical outcomes and fecal microbiome in active Crohn's disease. Inflammatory Bowel Diseases. 2015;21:556-563. DOI: 10.1097/MIB.0000000000000307

[82] Suskind DL, Singh N, Nielson H, Wahbeh G. Fecal microbial transplant via nasogastric tube for active pediatric ulcerative colitis. Journal of Pediatric Gastroenterology and Nutrition. 2015;60:27-29. DOI: 10.1097/MPG.0000000000000544

[83] Cui B, Feng Q, Wang H, Wang M, Peng Z, Li P, et al. Fecal microbiota transplantation through mid-gut for refractory Crohn's disease: Safety, feasibility, and efficacy trial results. Journal of Gastroenterology and Hepatology. 2015;30:51-58. DOI: 10.1111/jgh. 12727

[84] Torre LA, Bray F, Siegel RL, Ferlay J, Lortet-Tieulent J, Jemal A. Global cancer statistics, 2012. CA: A Cancer Journal for Clinicians. 2015;65:87-108. DOI: 10.3322/caac. 21262

[85] Sun J, Kato I. Gut microbiota, inflammation and colorectal cancer. Genes \& Diseases. 2016;3:130-143. DOI: 10.1016/j.gendis.2016.03.004

[86] Grivennikov SI. Inflammation and colorectal cancer: Colitis-associated neoplasia. Seminars in Immunopathology. 2013;35:229-244. DOI: 10.1007/s00281-012-0352-6

[87] Arthur JC, Gharaibeh RZ, Mühlbauer M, Perez-Chanona E, Uronis JM, McCafferty J, et al. Microbial genomic analysis reveals the essential role of inflammation in bacteria-induced colorectal cancer. Nature Communications. 2014;5:4724. DOI: 10.1038/ ncomms5724

[88] Robles AI, Traverso G, Zhang M, Roberts NJ, Khan MA, Joseph C, et al. Whole-exome sequencing analyses of inflammatory bowel disease-associated colorectal cancers. Gastroenterology. 2016;150:931-943. DOI: 10.1053/j.gastro.2015.12.036

[89] Nosho K, Sukawa Y, Adachi Y, Ito M, Mitsuhashi K, Kurihara H, et al. Association of Fusobacterium nucleatum with immunity and molecular alterations in colorectal cancer. World Journal of Gastroenterology. 2016;22:557-566. DOI: 10.3748/wjg.v22.i2.557

[90] Yu J, Feng Q, Wong SH, Zhang D, Liang QY, Qin Y, et al. Metagenomic analysis of faecal microbiome as a tool towards targeted non-invasive biomarkers for colorectal cancer. Gut. 2017;66:70-78. DOI: 10.1136/gutjnl-2015-309800

[91] Meira LB, Bugni JM, Green SL, Lee C-W, Pang B, Borenshtein D, et al. DNA damage induced by chronic inflammation contributes to colon carcinogenesis in mice. The Journal of Clinical Investigation. 2008;118:2516-2525. DOI: 10.1172/JCI35073

[92] Grivennikov SI, Wang K, Mucida D, Stewart CA, Schnabl B, Jauch D, et al. Adenomalinked barrier defects and microbial products drive IL-23/IL-17-mediated tumour growth. Nature. 2012;491:254-258. DOI: 10.1038/nature11465 
[93] Round JL, Mazmanian SK. The gut microbiota shapes intestinal immune responses during health and disease. Nature Reviews. Immunology. 2009;9:313-323. DOI: 10.1038/ nri2515

[94] Purcell RV, Visnovska M, Biggs PJ, Schmeier S, Frizelle FA. Distinct gut microbiome patterns associate with consensus molecular subtypes of colorectal cancer. Scientific Reports. 2017;7:11590. DOI: 10.1038/s41598-017-11237-6

[95] Prorok-Hamon M, Friswell MK, Alswied A, Roberts CL, Song F, Flanagan PK, et al. Colonic mucosa-associated diffusely adherent afaC+Escherichia coli expressing lpfA and pks are increased in inflammatory bowel disease and colon cancer. Gut. 2014;63:761770. DOI: 10.1136/gutjnl-2013-304739

[96] Jiménez B, Mirnezami R, Kinross J, Cloarec O, Keun HC, Holmes E, et al. 1H HR-MAS NMR spectroscopy of tumor-induced local metabolic "field-effects" enables colorectal cancer staging and prognostication. Journal of Proteome Research. 2013;12:959-968. DOI: $10.1021 / p r 3010106$

[97] Veselkov KA, Mirnezami R, Strittmatter N, Goldin RD, Kinross J, Speller AVM, et al. Chemo-informatic strategy for imaging mass spectrometry-based hyperspectral profiling of lipid signatures in colorectal cancer. Proceedings of the National Academy of Sciences of the United States of America. 2014;111:1216-1221. DOI: 10.1073/ pnas.1310524111

[98] Kinross J, Mirnezami R, Alexander J, Brown R, Scott A, Galea D, et al. A prospective analysis of mucosal microbiome-metabonome interactions in colorectal cancer using a combined MAS 1HNMR and metataxonomic strategy. Scientific Reports. 2017;7:8979. DOI: 10.1038/s41598-017-08150-3

[99] Wu Q, Yang Z-P, Xu P, Gao L-C, Fan D-M. Association between Helicobacter pylori infection and the risk of colorectal neoplasia: A systematic review and meta-analysis. Colorectal Disease: The Official Journal of the Association of Coloproctology of Great Britain and Ireland. 2013;15:e352-e364. DOI: 10.1111/codi.12284

[100] Halfvarson J, Brislawn CJ, Lamendella R, Vázquez-Baeza Y, Walters WA, Bramer LM, et al. Dynamics of the human gut microbiome in inflammatory bowel disease. Nature Microbiology. 2017;2:17004. DOI: 10.1038/nmicrobiol.2017.4

[101] Pascal V, Pozuelo M, Borruel N, Casellas F, Campos D, Santiago A, et al. A microbial signature for Crohn's disease. Gut. 2017;66:813-822. DOI: 10.1136/gutjnl-2016-313235

[102] Forbes JD, Van Domselaar G, Bernstein CN. Microbiome survey of the inflamed and noninflamed gut at different compartments within the gastrointestinal tract of inflammatory bowel disease patients. Inflammatory Bowel Diseases. 2016;22:817-825. DOI: 10.1097/MIB.0000000000000684

[103] Morgan XC, Tickle TL, Sokol H, Gevers D, Devaney KL, Ward DV, et al. Dysfunction of the intestinal microbiome in inflammatory bowel disease and treatment. Genome Biology. 2012;13:R79. DOI: 10.1186/gb-2012-13-9-r79 
[104] Santoru ML, Piras C, Murgia A, Palmas V, Camboni T, Liggi S, et al. Cross sectional evaluation of the gut-microbiome metabolome axis in an Italian cohort of IBD patients. Scientific Reports. 2017;7:1-14. DOI: 10.1038/s41598-017-10034-5

[105] Nishino K, Nishida A, Inoue R, Kawada Y, Ohno M, Sakai S, et al. Analysis of endoscopic brush samples identified mucosa-associated dysbiosis in inflammatory bowel disease. Journal of Gastroenterology. 2017. DOI: 10.1007/s00535-017-1384-4

[106] Marchesi JR, Dutilh BE, Hall N, Peters WHM, Roelofs R, Boleij A, et al. Towards the human colorectal cancer microbiome. PLoS One. 2011;6:e20447. DOI: 10.1371/journal. pone.0020447

[107] Zackular JP, Rogers MAM, Ruffin MT, Schloss PD. The human gut microbiome as a screening tool for colorectal cancer. Cancer Prevention Research (Philadelphia, PA). 2014;7:1112-1121. DOI: 10.1158/1940-6207.CAPR-14-0129

[108] Mima K, Cao Y, Chan AT, Qian ZR, Nowak JA, Masugi Y, et al. Fusobacterium nucleatum in colorectal carcinoma tissue according to tumor location. Clinical and Translational Gastroenterology. 2016;7:e200. DOI: 10.1038/ctg.2016.53

[109] Wei Z, Cao S, Liu S, Yao Z, Sun T, Li Y, et al. Could gut microbiota serve as prognostic biomarker associated with colorectal cancer patients' survival? A pilot study on relevant mechanism. Oncotarget. 2016;7:46158-46172. DOI: 10.18632/oncotarget.10064

[110] Alexander J, Perdones-Montero A, Scott A, Poynter L, Atkinson S, Soucek P, et al. OC-061 a prospective multi-national study of the colorectal cancer mucosal microbiome reveals specific taxonomic changes indicative of disease stage and prognosis. Gut. 2017;66:A32. DOI: 10.1136/gutjnl-2017-314472.61

[111] White JR, Drewes J, Sears CL. Abstract 844: High-resolution microbiome profiling and meta-analysis yields insight into microbial consortia associated with colorectal cancer. Cancer Research. 2016;76:844-844. DOI: 10.1158/1538-7445.AM2016-844 